\title{
AVALIAÇÃO DA QUALIDADE DE VIDA, ESTRESSE E SAÚDE MENTAL DOS PROFISSIONAIS DE SAÚDE DAS UNIDADES DE TERAPIA INTENSIVA
}

\author{
ASSESSMENT OF QUALITY OF LIFE, STRESS AND MENTAL \\ HEALTH OF HEALTH PROFESSIONALS IN INTENSIVE THERAPY \\ UNITS
}

\author{
Kelly Patrícia Medeiros Falcão Pascoal ${ }^{1}$ \\ Andréa Carla Brandão da C. Santos ${ }^{2}$ \\ Jullye Anne Sousa Siqueira da Silva ${ }^{3}$ \\ Vanessa Michelle de Souza Fernandes ${ }^{4}$ \\ Maria Nelusia de Sousa ${ }^{5}$
}

RESUMO: OBJETIVO: Avaliar a qualidade de vida, estresse e a saúde mental dos profissionais de saúde das UTI's. MÉTODO: Caracteriza-se como uma pesquisa de caráter transversal, descritiva e analítica, sendo um estudo de campo, com abordagem quantitativa. A pesquisa foi realizada no Hospital Municipal Santa Isabel, localizado em João Pessoa no período de abril de 2018. A população foi composta pelos profissionais de saúde que exercem sua função nas Unidades de Terapia Intensiva da referida unidade hospitalar. A amostra foi do tipo não-probabilística, composta por 6 fisioterapeutas, 5 médicos, 7 enfermeiros e 12 técnicos de enfermagem, totalizando 30 profissionais. Foram utilizados três instrumentos: Whoqol-Bref para avaliar a qualidade de vida; Self Report Questionaire (SRQ-20) para avaliar sofrimento mental; e Job Stress Scale (JSS) para avaliar o estresse no ambiente de trabalho. $O$ estudo seguiu os princípios éticos e legais, sendo aprovado pelo Comitê de Ética em Pesquisa sob protocolo $n^{\circ}$ 54/2018 e CAAE: 85321818.1.0000.5176. Os dados foram registrados na forma de banco de dados para o programa SPSS e analisados por meio da estatística descritiva, através das

\footnotetext{
1 Fisioterapeuta. Mestre em Ciências da Saúde pela UFPE. Especialista em Fisioterapia Cardio Respiratória pelo Centro Universitário de Joao Pessoa (UNIPÊ).

2 Fisioterapeuta. Doutora em Saúde Materno Infantil pelo IMIP. Docente do curso de fisioterapia do Centro Universitário de Joao Pessoa (UNIPÊ).

${ }^{3}$ Fisioterapeuta. Residente em Atenção ao Paciente Critico no HULW. Especialista em Fisioterapia em Unidade de Terapia Intensiva (UNIPÊ).

${ }^{4}$ Fisioterapeuta. Residente em Atenção ao Paciente Critico no HULW. Especialista em Fisioterapia em Unidade de Terapia Intensiva (UNIPÊ).

5 Fisioterapeuta. Coordenadora do serviço de fisioterapia do Hospital Municipal Santa Isabel. Especialista em Traumato-ortopedia.
} 
medidas de tendência central e de variabilidade, bem como a distribuição absoluta e relativa. A simetria das distribuições contínuas foi avaliada pelo teste de Kolmogorov-Smirnov, foi utilizada a Análise de Variância (ANOVA) para permitir identificar prováveis diferenças entre os grupos e para a interpretação das informações foi considerado um nível de significância de $5 \% \quad(p<0,05)$. RESULTADOS: A amostra apresentou prevalência do sexo feminino (66,7\%), média de idade de $37( \pm 8,15)$ anos. Houve predomínio de atividades em ambos os turnos, com tempo médio de atuação na área de 11,9 anos $( \pm 8,06)$. Analisando os dados referentes ao questionário Job Stresse Scale (JSS) houve predominância $(46,66 \%)$ do trabalho ativo, no Self-Reporting (SRQ) os participantes não apresentaram risco para desenvolvimento de Transtorno Mental Comum (TMC), a qualidade de vida dos participantes foi classificada como regular em todos os domínios do Whoqol-Bref. CONCLUSÃO: Neste sentido fica claro a necessidade de aprofundamento no tema em questão, a fim de identificar os fatores que levam a deterioração da qualidade de vida destes profissionais.

Palavras chave: Qualidade de vida. Saúde mental. Unidade de terapia intensiva.

ABSTRACT: OBJECTIVE: To evaluate the quality of life, stress and mental health of health professionals of the ICUs. METHOD: It is characterized as a cross-sectional, descriptive and analytical research, being a field study, with a quantitative approach. The research was carried out at Hospital Santa Isabel, located in João Pessoa during April / 2018. The population was composed by the health professionals who perform their function in the Intensive Care Units of said hospital unit. The sample was of non-probabilistic type, composed of 6 physiotherapists, 5 physicians, 7 nurses and 12 nursing technicians, totaling 30 professionals. Three instruments were used, the Whoqol-Bref to assess quality of life; the Self Report Questionnaire (SRQ-20) to assess mental distress; and the Job Stress Scale (JSS) to assess stress in the workplace. The study followed the ethical and legal principles, being approved by the Committee of Ethics in Research under protocol No. 54/2018 and CAAE: 85321818.1.0000.5176. The data were recorded in the form of SPSS computer program database and analyzed through descriptive statistics, through measures of central tendency and variability, as well as absolute and relative distribution. The symmetry of the continuous distributions was evaluated by the Kolmogorov-Smirnov test, the Analysis of Variance (ANOVA) was used to identify if there were differences between the groups and for the interpretation of the information at a significance level of 5\% ( $p$ <0.05). RESULTS: The sample had a female prevalence (66.7\%), mean age 37 ( \pm 8.15) years. There was a predominance of activities in both shifts, with average time in the area of 11.9 years $( \pm 8.06)$. Analyzing data on the Job Stress Scale (JSS) questionnaire, there was a predominance $(46.66 \%)$ of the active work, in the Self-Reporting (SRQ) participants presented in the risk for the development of Common Mental Disorder (CMD), quality of life of participants was classified as regular in all Whoqol-Bref domains. CONCLUSION: In this sense it is clear the need to deepen the theme in question, in order to identify the factors that lead to deterioration in the quality of life of these professionals.

Keywords: Quality of life. Mental health Intensive care unit. 


\section{INTRODUÇÃO}

O trabalho é considerado um dos fundamentos mais relevantes e significativos na vida de um indivíduo, sendo indissociável de sua própria existência, sendo também encarado como um meio de inserção do indivíduo na sociedade, englobando uma gama de aspectos físicos e psíquicos relacionados. O trabalho é, ainda, responsável por garantir a subsistência do trabalhador e de sua família, sendo um construtor de identidade, entretanto pode adquirir um caráter nocivo e patogênico ou ser uma fonte produtora de agravos à saúde mental e física (MOREIRA et al., 2017).

Com as atuais formas de organização do trabalho e a reestruturação produtiva, constatou-se enorme incremento da produtividade e intensificação deste, tornando o ambiente de trabalho gerador de novos riscos, resultando em maior exigência e sobrecarga para os profissionais. As mudanças se caracterizam por aumento do ritmo de trabalho, longas jornadas, pressão de tempo, repetitividade e monotonia das tarefas, conflitos interpessoais, isolamento social, ausência do poder de decisão, bem como maior controle da força de trabalho. Sendo assim, as exigências de trabalho, atreladas às condições individuais do trabalhador, podem repercutir negativamente em sua saúde física e mental (BAHIA, 2014).

Tais mudanças no universo do trabalho também têm exercido influência sobre as instituições hospitalares. Dessa forma, observa-se uma crescente preocupação em relação aos efeitos causados pelo trabalho nos profissionais de saúde (OLIVEIRA et al., 2006).

O sofrimento psíquico atrelado, ao trabalho no ambiente hospitalar, pode afetar a todos os profissionais, gerando quadros de somatização, absenteísmo, e o desenvolvimento de transtornos mentais, como ansiedade e depressão. O esgotamento emocional, a responsabilidade de cuidar, o medo de cometer erros, o cansaço, a dificuldade das relações estabelecidas com a equipe multidisciplinar, culminam em um estado de penosidade ocupacional, com repercussões psicológicas 
significativas no indivíduo, bem como na sua relação com os pacientes, gerando sentimentos de insatisfação para o profissional e clientes externos (familiares e pacientes) (LUCCHESI et al., 2008).

Além disso, Santana et al. (2014) afirmam que a equipe de saúde no ambiente hospitalar está frequentemente exposta a fatores que comprometem sua saúde física e mental, a exemplo da proximidade com situações de dor, sofrimento e morte, além do sistema de turnos ininterruptos de revezamento e prestação de serviços durante 24 horas, sete dias da semana, associado a transição entre turnos para passagem do plantão.

A Unidade de Terapia Intensiva (UTI), enquanto setor das instituições hospitalares constitui-se em um espaço de concentração de tecnologias duras que possibilitam a equipe de saúde na manutenção do quadro clínico dos pacientes e intervirem em casos de alterações hemodinâmicas e ventilatórias. Recursos tecnológicos como monitores, ventiladores mecânicos, bombas de infusão e oxímetros, com seus alarmes visuais e sonoros, são imprescindíveis para o processo de trabalho, alertando a equipe sobre as alterações clínicas do paciente e inadequado funcionamento dos próprios aparelhos (OLIVEIRA, SOUZA, 2012).

O trabalho diário dos profissionais de saúde intensivistas, exige conhecimento técnico qualificado, habilidades, atenção, raciocínio rápido e controle emocional para lidar com as adversidades, além de atualização científica contínua, tendo em vista o desenvolvimento dessa especialidade ao longo dos últimos anos. Consequentemente, a UTI é considerada por diversos autores como o ambiente mais estressante do hospital, o que contribui para o sofrimento psíquico dos profissionais de saúde que atuam nesse ambiente (ROCHA, CARVALHO, 2017).

Diante de tais pressupostos, o presente trabalho teve como objetivo avaliar a qualidade de vida, saúde mental e o estresse dos profissionais de saúde atuantes em UTI. Justificando-se pela necessidade da identificação destes aspectos, a fim de reduzir os danos causados aos profissionais de saúde no ambiente de trabalho, bem como, possibilitar a criação ou potencialização de estratégias para enfrentamento aos agentes estressores identificados. 


\section{MÉTODOS}

Caracterizou-se como um estudo de caráter transversal, descritivo e analítico, sendo um estudo de campo com abordagem quantitativa, realizado nas UTIs do Hospital Municipal Santa Isabel, localizado em João Pessoa, durante o mês de abril de 2018. O estudo seguiu os princípios éticos e legais, de acordo com as recomendações da Resolução $n^{\circ}$ 466/12 do CNS, sendo aprovado pelo Comitê de Ética em Pesquisa sob protocolo n ${ }^{\circ}$ 54/2018 e CAAE: 85321818.1.0000.5176.

A população foi composta pelos profissionais de saúde que exercem sua função nas UTIs da referida unidade hospitalar. A amostra foi do tipo nãoprobabilística, composta por 30 profissionais, sendo 6 fisioterapeutas, 5 médicos, 7 enfermeiros e 12 técnicos de enfermagem. Foram incluídos na pesquisa profissionais da área de saúde que trabalhavam há, pelo menos, 6 meses nas UTls de forma direta na assistência, com idade acima de 18 anos e excluídos os profissionais que não trabalhavam nas unidades de terapia intensiva diretamente na assistência e/ou não tinham o tempo mínimo necessário de atuação.

A coleta dos dados ocorreu a partir da aplicação de três instrumentos validados no Brasil, o Whoqol-Bref para avaliar a qualidade de vida e o Job Stress Scale (JSS) para avaliar o estresse no ambiente de trabalho e o Self Reporting Questionnaire (SRQ) para avaliar a saúde mental. Para complementar os dados foi utilizado um questionário feito pelos próprios pesquisadores, para identificar o perfil sócio demográfico dos profissionais.

Os dados foram registrados na forma de banco de dados do programa de informática SPSS (Statistical Package for Social Sciences) para Windows, versão 20.0, e analisados por meio da estatística descritiva, através das medidas de tendência central (média ou mediana) e de variabilidade (desvio padrão), bem como a distribuição absoluta e relativa. A simetria das distribuições contínuas foi avaliada pelo teste de Kolmogorov-Smirnov. Foi utilizada a Análise de Variância (ANOVA), para permitir identificar se havia diferenças entre os grupos. Ressalta-se, por fim, 
que para a interpretação das informações, foi considerado um nível de significância de $5 \%(p<0,05)$.

\section{RESULTADOS E DISCUSSÃO}

A amostra deste estudo foi constituída de 30 profissionais de saúde que foram avaliados quanto aos aspectos sociodemográficos e laborais, quanto ao estresse no ambiente de trabalho, saúde mental e qualidade de vida.

Analisando os resultados observou-se prevalência do sexo feminino, correspondendo a $66,7 \%$ da amostra $(n=20)$, enquanto $33,3 \%(n=10)$ correspondiam a indivíduos do sexo masculino. Quanto a predominância do sexo, sabe-se que há muitas décadas o setor saúde é, estrutural e historicamente, feminino, fato que se relaciona, provavelmente, à própria essência da profissão, o cuidado, visto que o ato de cuidar sempre foi associado à figura feminina. Apesar disso, a literatura destaca um discreto aumento da participação masculina no setor, principalmente na enfermagem (BORGES; BIANCHIN, 2015; MACHADO et al., 2015).

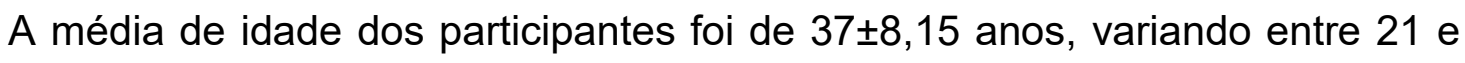
54 anos. Araújo et al. (2017) afirmam que toda a vivência acumulada ao longo dos anos concorre para o desenvolvimento de suas atribuições com maior segurança e agilidade. No entanto, sabe-se que a juventude é vista como sinônimo de vida, força e criatividade, de modo que o profissional jovem passa a ser aceito como flexível, à medida que algumas instituições de saúde acreditam na possibilidade de se adequar mais facilmente esses profissionais as filosofias institucionais.

Quanto às características laborais, 40\% (n=12) dos participantes eram técnicos de enfermagem, 23,3\% $(n=7)$ eram enfermeiros, 20\% $(n=6)$ eram fisioterapeutas e 16,7\% $(n=5)$ eram médicos. Desses, 70\% $(n=21)$ desenvolviam suas atividades em ambos os turnos, $20 \%(n=6)$ no período diurno e $10 \%(n=3)$ no período noturno.

Segundo Guerra et al. (2016), o trabalho quando desenvolvido em regime de turnos pode favorecer a maior sonolência diurna e a diminuição do estado de alerta 
do indivíduo, expondo-o a um maior risco de acidentes de trabalho. Além disso, esse regime de turnos associado ao trabalho com pacientes críticos, pode gerar impacto em várias dimensões da qualidade de vida dos profissionais de saúde.

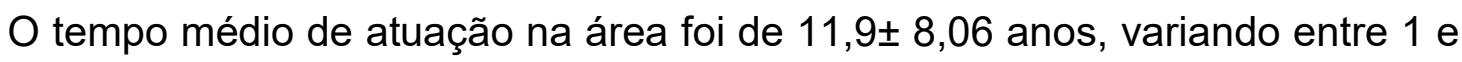
28 anos. Preto; Pedrão (2014) destacam que essa variação dos anos representa um fator favorável para o bom desempenho profissional, à medida que os profissionais com menos experiência têm oportunidade de discutir suas dificuldades com os profissionais que apresentam maior experiência, que provavelmente dividem 0 mesmo espaço.

Analisando os dados referentes ao JSS (Job Stress Scale), que avalia as características psicológicas e sociais do trabalho, através das dimensões demanda psicológica, controle e apoio social, classificou-se os participantes segundo modelo Demanda-controle de karasek.

Consequentemente, os participantes foram classificados de acordo com o tipo de trabalho desempenhado em: trabalho ativo, trabalho passivo, alto desgaste e baixo desgaste. Para tanto, foi instituído um ponto de corte em função da mediana obtida para cada dimensão avaliada, e os participantes foram classificados em dois grupos, os que ficaram com escore igual ou acima da mediana como "alto" e abaixo da mediana como "baixo". Foram classificados como "alta demanda" os escores $\geq 15$ pontos, "baixa demanda" $\leq 14$ pontos, "alto controle" os escores $\geq 20$ pontos e "baixo controle" $\leq 19$ pontos (Tabela 1). 
Tabela 1 - Exposição dos resultados segundo modelo Demanda-Controle de Karasek.

\begin{tabular}{lcc}
\hline & N & $\%$ \\
\hline Trabalho ativo & 14 & 46,66 \\
Trabalho passivo & 3 & 10 \\
Alto desgaste & 9 & 30 \\
Baixo desgaste & 4 & 13,34 \\
\hline
\end{tabular}

Fonte: Acervo do autor, 2018.

Analisando os dados, pode-se observar uma predominância $(46,66 \%)$ do trabalho ativo entre os profissionais de saúde estudados.

São considerados ativos os trabalhos que possuem altas demandas psicológicas, no entanto, permitem ao trabalhador decidir como e quando desenvolver suas tarefas, bem como usar seu potencial intelectual para isso. Dessa forma, se associa a desfechos psicológicos positivos, como aprendizado e crescimento pessoal, aliados a alta produtividade (FEIJÓ et al., 2017; SCHMIDT, 2013).

O estudo atual apresentou resultado semelhante ao desenvolvido por Olhôa et al. (2010), onde analisou 136 profissionais de Unidades de Terapia Intensiva (médicos, enfermeiros e técnicos de enfermagem) em um Hospital municipal, encontrando predominância do Trabalho Ativo entre eles.

Referente ao apoio social, os participantes foram classificados em alto $(\geq 19$ pontos) e baixo ( $\leq 18$ pontos) de acordo com a mediana obtida. Foi encontrado que $53,36 \%(n=16)$ da amostra obtiveram alto apoio social e 46,64 \% $(n=14)$ apresentaram baixo apoio social.

Feijó et al. (2017) afirmam que quanto maior o apoio social, menores são as chances de o trabalhador apresentar repercussões negativas à saúde, no entanto, o baixo apoio social pode estar associado a ocorrência de transtornos mentais comuns.

Os dados referentes ao questionário Self-Reporting, demostraram uma média no valor de 4,73 $\pm 4,00$ pontos. Realizando o cálculo de acordo com sexo, se obteve média $4,8 \pm 3,84$ para o sexo feminino e $4,6 \pm 4,50$ para o sexo masculino. De acordo com o ponto de corte adequado (7/8 pontos), apresentado por Gonçalves et al. 
(2008), a média dos participantes de ambos os sexos não apresentou risco para o desenvolvimento de transtornos mentais comuns.

Bertoncello et al. (2017) afirmam que a saúde mental dos profissionais está diretamente relacionada com o suporte organizacional recebido por eles no ambiente de trabalho. Quanto maior o suporte, menor será o estresse ocupacional, resultando, consequentemente, em uma melhor saúde mental com menos Risco de Transtorno Mental Comum.

$\mathrm{Na}$ avaliação do questionário WHOQOL-BREF se obteve como média

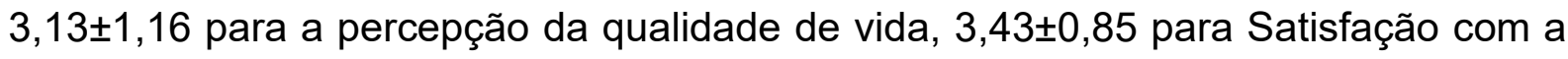

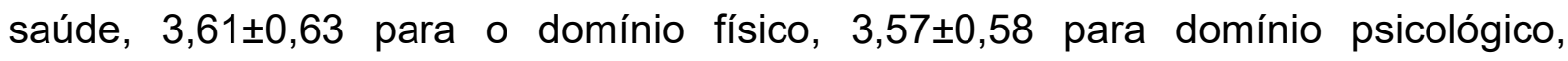

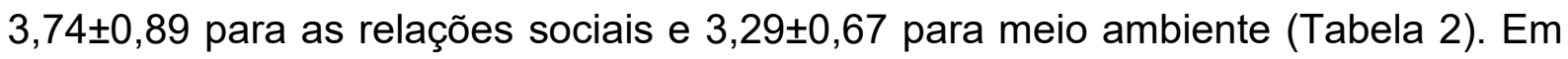
suma, os resultados apontam para uma qualidade de vida "regular" em todos os domínios do Whoqol-Bref, com base na média geral.

Tabela 2 - Resultados obtidos para os Domínios do Whoqol-Bref agrupados por profissão.

\begin{tabular}{lccccc}
\hline & Total & Fisioterapeuta & Enfermeiro & $\begin{array}{c}\text { Técnico de } \\
\text { Enfermagem }\end{array}$ & Médico \\
\hline $\begin{array}{l}\text { Percepção } \\
\text { da QV }\end{array}$ & $3,13 \pm 1,16$ & $3,33 \pm 1,03$ & $2,57 \pm 1,27$ & $3,25 \pm 1,28$ & $3,40 \pm 0,89$ \\
$\begin{array}{l}\text { Satisfação } \\
\text { com a saúde }\end{array}$ & $3,43 \pm 0,85$ & $3,33 \pm 0,81$ & $3,86 \pm 0,90$ & $3,17 \pm 0,93$ & $3,60 \pm 0,54$ \\
$\begin{array}{l}\text { Domínio } \\
\text { físico }\end{array}$ & $3,61 \pm 0,63$ & $3,7 \pm 0,56$ & $3,67 \pm 0,80$ & $3,58 \pm 0,69$ & $3,53 \pm 0,45$ \\
$\begin{array}{l}\text { Domínio } \\
\text { psicológico }\end{array}$ & $3,57 \pm 0,58$ & $3,38 \pm 0,67$ & $3,56 \pm 0,65$ & $3,59 \pm 0,59$ & $3,76 \pm 0,48$ \\
$\begin{array}{l}\text { Relações } \\
\text { sociais }\end{array}$ & $3,74 \pm 0,89$ & $3,82 \pm 0,83$ & $3,64 \pm 1,23$ & $3,77 \pm 0,88$ & $3,71 \pm 0,70$ \\
$\begin{array}{l}\text { Meio } \\
\text { ambiente }\end{array}$ & $3,29 \pm 0,67$ & $3,51 \pm 0,67$ & $3,39 \pm 0,65$ & $2,94 \pm 0,50$ & $3,72 \pm 0,84$ \\
\hline
\end{tabular}

Fonte: Acervo do autor, 2018.

Estes resultados divergem daqueles encontrados por Branco et al. (2010) em estudo semelhante, onde avaliou 365 profissionais de saúde do âmbito hospitalar, evidenciando uma qualidade de vida satisfatória em todos os domínios do WhoqolBref para eles. 
O mesmo autor destaca, ainda, que os gestores de recursos humanos devem buscar identificar quais aspectos da qualidade de vida estão comprometidos, podendo, assim, intervir nesses pontos, a fim de gerar maior satisfação desses profissionais, melhorando sua produtividade e, consequentemente, a qualidade da assistência prestada.

Ao se comparar os domínios de qualidade de vida entre os profissionais não foi encontrada diferença significativa $(p>0,05)$, porém, ao analisar as médias e desvios padrão (Tabela 2), observa-se diferença entre os grupos. De acordo com os dados há uma diferença na percepção da qualidade de vida do enfermeiro, classificada como "ruim" através da escala Likert, de acordo com a média geral obtida para o domínio. O estudo atual corrobora com os achados de Furtado, Pereira e Santos (2017), que avaliaram 61 profissionais de saúde atuantes em serviços de urgência, encontrando escore menor para a percepção da qualidade de vida nos profissionais de enfermagem.

Quirino et al. (2016) afirmam que essa alteração da percepção da qualidade vida pode estar relacionada ao fato de que em sua maioria, estes profissionais possuem mais de um vínculo empregatício, cumprindo carga horárias superiores ao recomendado, contribuindo para o seu desgaste físico e emocional.

Outra observação diz respeito ao domínio meio ambiente na percepção dos técnicos de enfermagem, que obteve classificação "ruim" através da escala Likert, de acordo com a média geral obtida para o domínio. Nesse domínio estão as questões relacionadas à segurança, condições do ambiente físico, remuneração, lazer, moradia, transporte e acesso aos serviços de saúde.

Tais resultados corroboram com o estudo de Paschoa, Zanei e Whitaker (2007), que avaliaram a qualidade de vida de 126 técnicos e auxiliares de enfermagem de UTI's, tendo por resultado a constatação de que as facetas que contribuíram para que este domínio obtivesse menor escore, foram oportunidades de lazer e a baixa remuneração para satisfação das necessidades. Além disto, o domínio Meio ambiente foi o que obteve menor escore em diversos estudos desenvolvidos com profissionais de saúde atuantes no serviço hospitalar (BRANCO et al., 2010; OLIVEIRA et al., 2016). 


\section{CONCLUSÃO}

A partir dos questionários analisados, foi observado que os profissionais das unidades de terapia analisadas, não apresentaram alto risco para o desenvolvimento de transtornos mentais comuns. Analisando o JSS pode-se perceber que a maioria dos profissionais de saúde das unidades desempenham trabalho do tipo "ativo", com níveis satisfatórios de apoio social. A qualidade de vida dos profissionais, expressa em médias, apresentou-se como "regular" para todos os domínios do Whoqol-Bref com pequenas variações dos escores nos domínios. Nesse sentido, fica evidente a necessidade de aprofundamento no tema em questão e o estudo da relação dessas variáveis com os diversos fatores que podem ser considerados estressores no ambiente das UTIs e na vida dos profissionais de saúde, tendo em vista a divergência de resultados descritos na literatura. A criação de novas abordagens direcionadas a esse público é essencial, a fim de identificar os fatores que levam a pior qualidade de vida, deterioração da saúde mental e estresse nestes profissionais, visto que tais alterações podem afetar diretamente a assistência prestada e sua convivência no ambiente familiar e social.

\section{REFERÊNCIAS BIBLIOGRÁFICAS}

ARAÚJO, M.A.N.; et al. Perfil sociodemográficos dos enfermeiros da rede hospitalar. Revista Enfermagem UFPE, v.11, n.11, 2017.

BAHIA. Secretaria da Saúde do Estado. Superintendência de Vigilância e Proteção da Saúde. Diretoria de Vigilância e Atenção à Saúde do Trabalhador. Centro Estadual de Referência em Saúde do Trabalhador. Protocolo de atenção à saúde mental e trabalho. Salvador: DIVAST, 2014.

BERTONCELLO, B.; et al. Relações entre Estresse, Saúde Mental e Suporte Organizacional em um Hospital de Ensino. Investigação Qualitativa em Saúde, v. 2, n.1, 2017.

BRANCO, J.C.; et al. Qualidade de vida de colaboradores de hospital universitário do Sul do Brasil. J Helth Sci Inst, v.28, n.2, 2010.

BORGES, T.; BIANCHIN, M.A. Qualidade de vida dos profissionais de enfermagem de um hospital universitário do interior de São Paulo. Arq ciênc Saúde, v.22, n.1, 2015.

FEIJÓ, F.R.; et al. Estresse ocupacional em trabalhadores de uma fundação de atendimento 
socioeducativo: prevalência e fatores associados. Revista Psic, v.10, n.33, 2017.

FURTADO, F.M.S.F.; PEREIRA, L.B.; SANTOS, M.F. Avaliação da qualidade de vida e satisfação com a saúde de profissionais do SAMU envolvidos em ocorrências de rua. CONBRACIS, v.1, 2017.

GUERRA, P.C.; OLIVEIRA, N.F.; TERRERI, M.T.R.A.; Sono, qualidade de vida e humor em profissionais de enfermagem em Unidades de Terapia Intensiva Infantil. Rev Esc Enferm, v.50, n.2, 2016.

LUCCHESI, F.; MACEDO, P. C. M.; MARCO, M. A. Saúde mental na unidade de terapia intensiva. Rev. SBPH, v. 11, n. 1, p. 19-30, 2008.

MACHADO, M.H.; et al. Características Gerais da Enfermagem: o perfil sócio demográfico. Enferm foco, v.7, n.1/4, p.11-17, 2015.

MOREIRA, I. J. B.; et al. Aspectos psicossociais do trabalho e sofrimento psíquico na estratégia de saúde da família. R. Epidemiol control infec, v.7, n. 1, 2017.

OLIVEIRA, E. B.; SOUZA, N. V. M. Estresse e inovação tecnológica em unidade de terapia intensiva de cardiologia: tecnologia dura. Rev.enferm, v.20, n.4, 2012.

OLIVEIRA, G.M., et al. Influência do turno de trabalho na qualidade de vida dos profissionais de enfermagem de um hospital público do noroeste do Mato Grosso- MT. Rev. Cient. Sena Aires, v. 5, n. $1,2016$.

OLIVEIRA, P. R.; TRISTÃO, R. M.; NEIVA, E. R. Bornout e suporte organizacional em profissionais de UTI - Neonatal. Educação profissional: ciência e tecnologia, v.1, n.1, 2006.

PASHOA, S.; ZANEI, S.S.V.; IVETH, Y.W. Qualidade de vida dos trabalhadores de enfermagem de Unidades de terapia intensiva. Acta Paul Enferm, v.20, n.3, 2007.

PRETO, V.A.; PEDRÃO, L.J. A percepção de enfermeiros de Unidade de Terapia Intensiva sobre o estresse em seu local de trabalho. Rev enferm UFPE. V.8, n.9, 2014.

QUIRINO, G. S. Dupla jornada de trabalho: implicações na saúde da enfermeira. Revista de enfermagem UFPE, v.10, n.9, 2016.

ROCHA, G.S.; CARVALHO, R.C. Distúrbios Psíquicos menores em trabalhadores de UTI cadastrados na Associação Brasileira de Medicina Intensiva (AMIB). Disponível em: <http://www.sbpcnet.org.br/livro/68ra/resumos/resumos/4223_1adf8f80ffdd2aca9e3d34a85ea869 9f3.pdf>. Acesso em: 30 de novembro de 2017.

SANTANA, V. S.; et al. Qualidade de vida dos profissionais em ambiente hospitalar. Rev pesquisa em fisioterapia, v.4, n.1, 2014.

SCHMIDT, D. R. C. Modelo Demanda-Controle e estresse ocupacional entre profissionais de enfermagem: revisão integrativa. Rev. bras. enferm., v.66, n.5, p. 779-788, 2013.

ULHÔA, M.A.; et al. Distúrbios psíquicos menores e condições de trabalho em motoristas de caminhão. Rev saúde pública, v.44, n.6, 2010. 